\title{
COVALENT IMMOBILIZATION OF GLUCOSE OXIDASE ON WELL-DEFINED POLYMER-SILICON WAFER HYBRIDS VIA SURFACE-INITIATED RAFT-MEDIATED PROCESS
}

\author{
JIAYOU JI, KEQIANG XIA, TINGTING LIU, AND LIANG LI* \\ School of Materials Science and Engineering, Wuhan Institute of Technology, Wuhan 430073, P. R. China
}

(Received: May 30, 2012 - Accepted: June 3, 2013)

\begin{abstract}
A surface modification technique was developed for the functionalization of silicon surface with glucose oxidase (GOD). The silicon surface was first graft copolymerized with glycidyl methacrylate (GMA) via surface-initiated reversible addition-fragmentation chain-transfer (RAFT)-mediated process. GOD was then covalently immobilized through the ring-opening reaction between the amine groups of the GOD and the epoxide groups of the grafted GMA polymer chains. X-ray photoelectron spectroscopy (XPS) was used to characterize the surface-modified surface after each modification stage. Increasing the thickness of the polymer layer and the immobilization time could allow a great amount of GOD to be immobilized on the silicon surface. The GOD-functionalized silicon hybrids are promising candidates for the silicon-based glucose biosensors.
\end{abstract}

Keywords: surface-initiated RAFT, silicon, poly(glycidyl methacrylate), glucose oxidase.

\section{INTRODUCTION}

Silicon-based biomedical microdevices play an important role in biomedical engineering and biotechnology. ${ }^{1-4}$ It is of great importance to improve the biocompatibility of the silicon microdevices and to develop silicon surfaces with controllable cell adhesion. Immobilization of biomolecules on silicon substrates can be achieved via many methods such as adsorption, entrapment, covalent binding, etc. Functionalization of silicon surface with organics is a key in the development of new silicon-based biomedical devices. Tethering of polymer brushes on the solid substrate is an effective method of modifying the surface of the various substrates. ${ }^{5-6}$ The dense polymer brushes can alter the properties of the surface, supply excellent mechanical and chemical protection to the substrate, and indicate new pathways to the functionalization of silicon surfaces for molecular recognition and sensing. ${ }^{7-10}$

Recent progress in the techniques of polymer synthesis makes it possible to produce well-defined polymer chains on the surfaces of various substrates. Living free-radical polymerizations, such as nitroxide-mediated radical polymerization, ${ }^{11}$ atom transfer radical polymerization (ATRP) ${ }^{12}$ and reversible addition-fragmentation chain-transfer (RAFT) ${ }^{13}$ polymerizations, open up a promising way of designing and controlling macromolecular architecture under mild reaction condition. ATRP has been successfully used to synthesize the well-defined polymer-silicon hybrids. ${ }^{14-18}$ Reversible addition-fragmentation chain-transfer (RAFT)-mediated polymerization is an alternative method in the controlled free-radical polymerization. It involves a reversible addition-fragmentation cycle where the transfer of a dithioester moiety between the active and dormant species maintains the controlled feature of the polymerization process. ${ }^{19-21}$ Thus, it is expected that polymerization by the RAFT-moderated process can be used to produce well-defined side chains. The well-defined side chains with controlled length and chain architecture, and narrow molecular weight distribution, are particularly interesting for the field of biomedical polymers. Relatively few studies have been reported to the modification of silicon surfaces by the RAFT process and the application in biotechnology. ${ }^{13,22,23}$

Herein, well-defined polymer-silicon wafer hybrids, tethered brushes of poly(glycidyl methacrylate) (PGMA) on a silicon wafer, were prepared via surface-initiated RAFT living radical polymerization. Kinetics study on the surface-initiated RAFT of GMA revealed that the chain growth from the silicon surface was consistent with a "controlled" process. PGMA is a known surface linker and spacer for biomolecules. ${ }^{24,25}$ Then GOD was covalently immobilized on the modified silicon surface through the ring-opening reaction between the amine groups of the GOD and the epoxide groups of the grafted GMA polymer chains. The composition of the modified silicon surface was characterized by X-ray photoelectron spectroscopy (XPS). The dependence of the amount of immobilized GOD on the immobilized time and the thickness of grafted PGMA were also studied. The GOD-functionalized silicon hybrids could be promising candidates for the silicon-based glucose biosensors

\section{EXPERIMENTAL}

Materials

The silicon wafers were purchased from Unisil Co. (Santa Clara, CA,
USA). The silicon wafers were cut into square chips of about $1.2 \mathrm{~cm} \times 1.2$ $\mathrm{cm}$ in size. The monomer, GMA, was passed through inhibitor-removing columns and then stored under an argon atmosphere at $-10{ }^{\circ} \mathrm{C}$. The initiator a,a'-azobis(isobutyronitrile) (AIBN, 97\%) was obtained from Kanto Chemical Co. (Tokyo, Japan) and was recrystallized in anhydrous ethanol. The silane coupling agent (3-(trimethoxysilyl) propyl methacrylate (TMSPM, 98\%)) was obtained from Aldrich Chemical Co. (St. Louis, MO, USA). The chain transfer agent (CAT), 1-phenylethyl dithiobenzoate (PDB), was synthesized according to the published procedures. ${ }^{19}$ GOD (Type II, 15500 units $\mathrm{g}^{-1}$ from Aspergillus niger) was purchased from Sigma Chemical Co. (St. Louis, MO, USA). Dulbecco's phosphate buffer solution (PBS, $\mathrm{pH}=7.4$ ) was freshly prepared. Bio-Rad dye reagent for protein assay (Catalog No. 500-0006) was obtained from BioRad (Hercules, CA, USA). The other reagents were of analytical grade and were used without further purification unless otherwise mentioned.

\section{Immobilization process}

The process of surface functionalization of silicon was shown in Fig. 1. Coupling of the RAFT initiator to the silicon wafer was first achieved by adding TMSPM $(0.41 \mathrm{mmol})$, AIBN $(0.23 \mathrm{mmol})$, and PDB $(0.46 \mathrm{mmol})$ at a molar ratio of 1.8:1:2 into $5 \mathrm{~mL}$ of dry DMF in a Pyrex tube containing the silicon chip. After purging the mixture with argon for $30 \mathrm{~min}$, the surface coupling reaction continued at $60^{\circ} \mathrm{C}$ for $24 \mathrm{~h}$ under an argon atmosphere. After the reaction, the modified substrate was washed with $\mathrm{CH}_{2} \mathrm{Cl}_{2}$ and then with an ethanol/water mixture.

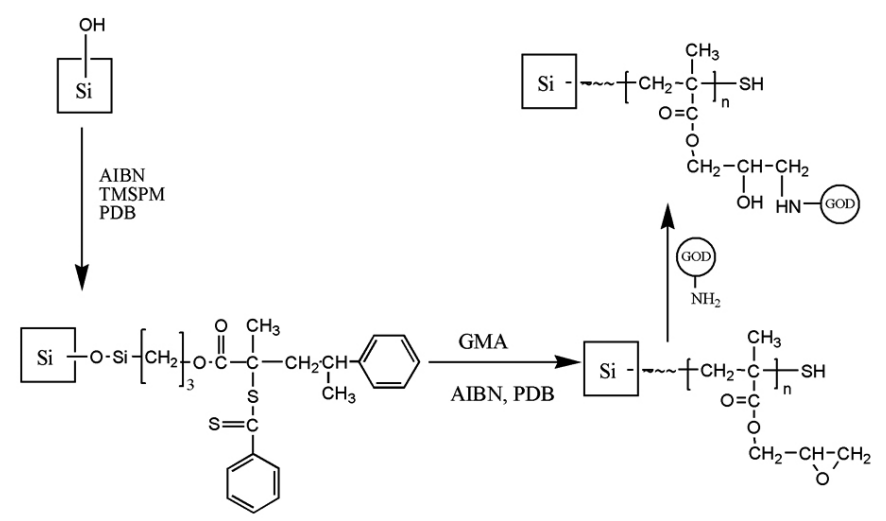

Fig. 1. Schematic diagram illustrating the process for the functionaliztion of the silicon surface.

For the preparation of PGMA brushes on the silicon wafer, the reaction conditions were as follows: [monomer]:[AIBN]:[PDB] molar feed ratio of 200:1:2 in $3 \mathrm{~mL}$ of dry DMF in a Pyrex tube containing the modified silicon chip. The solution was purged with argon for about $30 \mathrm{~min}$ to remove the dissolved oxygen. Then the tube was sealed and put in an oil bath at $60{ }^{\circ} \mathrm{C}$ for 
a predetermined period of time. After the reaction, the polymer-silicon wafer hybrids were extracted with THF, subsequently immersed in a large volume of their respective solvent system for $48 \mathrm{~h}$ to remove completely the adhered and physically adsorbed polymer.

The covalent immobilization of GOD onto the GMA grafted silicon surface was facilitated by the activation of the epoxide groups grafted on the surface. ${ }^{14}$ The Si-PGMA hybrids $(1 \mathrm{~cm} \times 1 \mathrm{~cm})$ were transferred to $3 \mathrm{~mL}$ of the $0.1 \mathrm{M}$ PBS (pH 7.4) containing GOD at a concentration of $4 \mathrm{mg} / \mathrm{ml}$. The immobilization was allowed to proceed under stirring at room temperature for a predetermined time. Then the reversibly bound GOD was desorbed in copious amounts of PBS for $24 \mathrm{~h}$ at room temperature.

\section{Characterization}

XPS analysis of the composites was carried out on a Kratos AXIS HSi spectrometer (Manchester, UK) with a monochromotized Al Ka X-ray source (1486.7 eV photons) and procedures similar to the reports before. ${ }^{26}$ The static water contact angles of the pristine and functionalized $\mathrm{Si}-\mathrm{H}$ surfaces were measured at $25{ }^{\circ} \mathrm{C}$ and $60 \%$ relative humidity on a telescopic goniometer (Rame-Hart model 10000-230, Moutain Lake, NJ, USA). For each angle reported, at least four measurements from different surface locations were averaged. The angle reported was reliable to $\pm 3^{\circ}$. The thickness of the polymer brushes grafted on the silicon substrate was determined by ellipsometry. The measurements were performed on a variable angle spectroscopic ellipsometer (M-vase, Woollam Inc., Lincoln, NE, USA) at incident angles of $70^{\circ}$ and $75^{\circ}$ in the wavelength range $200-1000 \mathrm{~nm}$. For each sample, the thickness measurements were made on at least three different surface locations.

The amount of GOD immobilized on the surface was determined by the modified dye-interaction methods ${ }^{27,28}$ with the BioRad protein dye reagent. For the preparation of the dye solution, the Bio-Rad stock dye solution was diluted five times with doubly-distilled water. GOD solution $(100 \mathrm{~mL})$ of known concentration was added to $5 \mathrm{~mL}$ of the dye solution. The GOD-dye solution was kept for $3 \mathrm{~h}$ and then centrifuged. The GOD-dye complexes were precipitated and the free dye remained in the upper layer. The absorbance of the supernatant at $465 \mathrm{~nm}$ was used for the standard calibration. For the quantitative determination of immobilized GOD, the dye solution $(5 \mathrm{~mL})$ was added to a test tube containing the Si-PGMA-GOD chip. After $3 \mathrm{~h}$ of reaction, the chip was removed and the absorbance of the dye solution was measured at $465 \mathrm{~nm}$. The amount of GOD immobilized on the chip was calculated based on the standard calibration. The activity of the immobilized GOD was deduced from the consumption rate of the b-D-(+)-glucose $(1.8 \mathrm{wt} \%)$ as the assay medium. The enzymatic reaction was initiated by the introduction of a Si-PGMAGOD chip $(1 \mathrm{~cm} \times 1 \mathrm{~cm})$ into the glucose solution at room temperature with agitation. The concentration of the b-D-(+)-glucose solution was measured on a biochemistry analyzer (YSI model 2700, Yellow Springs, OH, USA). The test was carried out 3 times for each sample.

\section{RESULTS AND DISCUSSION}

For the preparation of polymer brushes on the silicon surface, a uniform monolayer of initiators immobilized on the silicon surface is indispensable. Uniform silane layers can be coupled on $\mathrm{SiO}_{2}$ surfaces. ${ }^{29}$ Coupling of the RAFT initiator was achieved via a one-step process in the $\mathrm{SiO}_{2}$ region of the pristine $\mathrm{Si}$ wafer from reactions with a mixture of TMSPM (bearing a terminal double bond), AIBN, and PDB. The corresponding S $2 p$ core-level spectra of the pristine (oxide-covered) $\mathrm{Si}$ wafer and the PDB-Si surface were shown in Fig. 2. The $\mathrm{S} 2 p$ core-level spectrum of the PDB-Si surface consisted of the $\mathrm{S}$ $2 p 3 / 2$ and S $2 p 1 / 2$ peak components at the BE of about 163.9 and $165.1 \mathrm{eV}$, respectively, ${ }^{30}$ which indicated the presence of the RAFT initiator on the silicon surface. The static water contact angle increased from about $22^{\circ}$ for the pristine Si surface to about $57^{\circ}$ for the PDB-Si surface, which was consistent with the result of coverage of the Si surface by RAFT initiator by XPS.

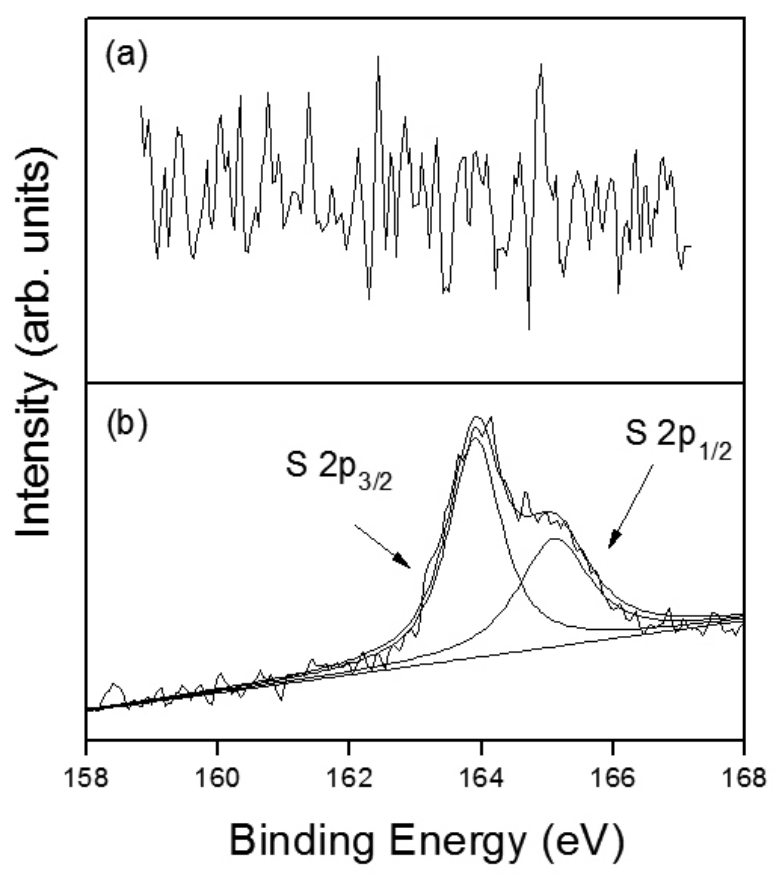

Fig. 2. S $2 p$ core-level spectra of (a) the pristine (oxide-covered) Si wafer and (b) the PDB-Si surface.

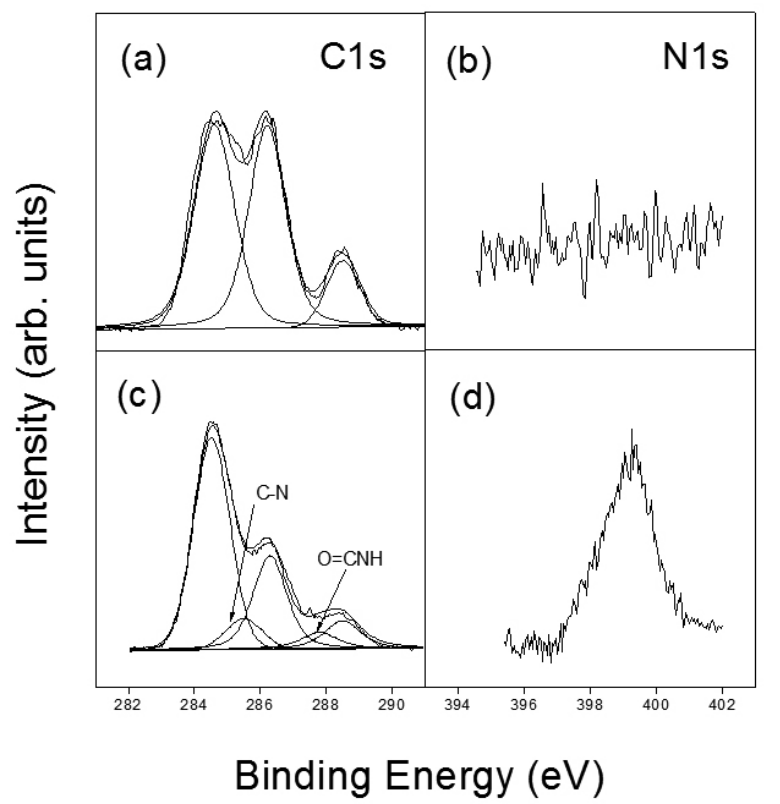

Fig. 3. $\mathrm{C} 1 s$ and $\mathrm{N} 1 s$ core-level spectra of $(\mathrm{a}, \mathrm{b})$ the Si-PGMA surface obtained at RAFT time of $20 \mathrm{~h}$ and $(\mathrm{c}, \mathrm{d})$ the Si-PGMA-GOD surface obtained at the GOD immobilized time of $5 \mathrm{~h}$.

The physicochemical properties of the silicon surface can be controlled and tuned by the choice of different vinyl monomers. In this work, glycidyl methacrylate (GMA) was chosen because its corresponding polymer is wellknown potential linkers for biomolecules..$^{24,25,31}$ As shown in C $1 s$ core-level spectra of the Si-PGMA surface (Fig. 3a), the presence of the grafted polymer on the silicon surface was ascertained by XPS analysis. The C $1 s$ core-level spectra can be curve-fitted into three peak components with BE at about 284.6 $\mathrm{eV}$ for $C-H$ species, $286.2 \mathrm{eV}$ for $C-\mathrm{O}$ species and $288.5 \mathrm{eV}$ for $\mathrm{O}=C$-O species, respectively. ${ }^{29,32}$ The $[\mathrm{C}-\mathrm{O}] /[\mathrm{O}=\mathrm{C}-\mathrm{O}]$ ratio obtained by XPS is about $3.1: 1$ and in agreement with its respective chemical structure of PGMA. In addition, 
when the silicon surface was grafted with PGMA, the water contact angle was tuned to $68^{\circ}$. The kinetics of PGMA growth from the Si-PDB surfaces via RAFT polymerization was investigated. An approximately linear increase in thickness of the grafted PGMA brushes on the surfaces with polymerization time was observed, as shown in Fig. 4. The results suggested that the chain growth from the Si-PDB surface was consistent with a "controlled" process.

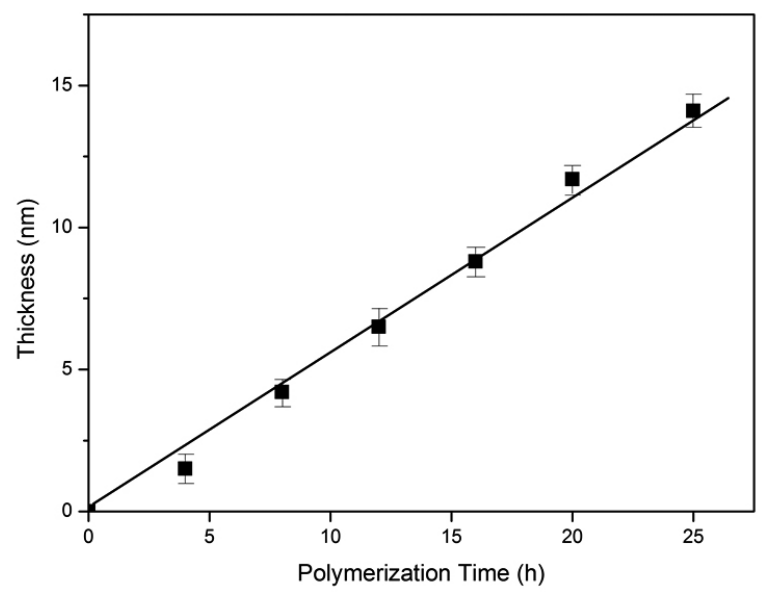

Fig. 4. Dependence of the grafted polymer film thickness on the RAFT polymerization time for the Si-PGMA surfaces. RAFT reaction conditions: [monomer]:[AIBN]:[PDB] $=200: 1: 2$ in dry DMF at $60{ }^{\circ} \mathrm{C}$ on chain transfer agent immobilized Si surface.

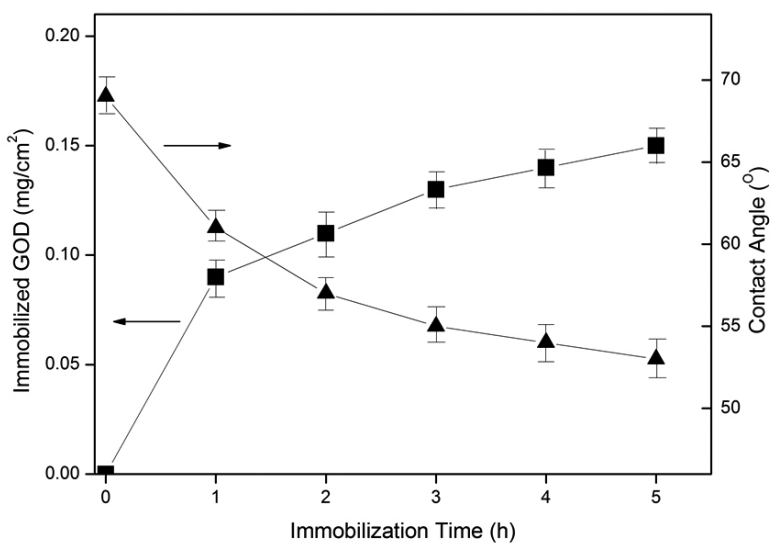

Fig. 5. Dependence of the amount of immobilized GOD and static water contact angle of the Si-PGMA-GOD surface on the immobilization time. The starting surface corresponds to the Si-PGMA surface obtained from $25 \mathrm{~h}$ of RAFT polymerization.

GOD was then covalently immobilized through the ring-opening reaction between the amine groups of the GOD and the epoxide groups of the grafted GMA polymer chains. After the removal of the reversibly bound GOD on the Si-PGMA-GOD surfaces, the C $1 s$ and N $1 s$ core-level spectra of the SiPGMA-GOD surfaces were compared to those of the Si-PGMA surface in Fig. 3. It was observed that the $\mathrm{C} 1 s$ and $\mathrm{N} 1 s$ spectra of the Si-PGMA-GOD surfaces were significantly different from the corresponding spectra of the $\mathrm{Si}$ PGMA surface. The C $1 s$ core-level spectra of the Si-PGMA-GOD surfaces obtained at the GOD immobilization time of $5 \mathrm{~h}$ (Fig. 3c) can be curve-fitted with five peak components having BE at about 284.6, 285.5, 286.2, 287.8, and $288.5 \mathrm{eV}$, attributable to the $C-H, C-N, C-O, O=C N H$, and $O=C-O$ species, respectively. ${ }^{30}$ The $C-N$ peak component is associated with the linkage in GOD and the linkage between PGMA and GOD as well. The $O=C N H$ peak component is associated with the peptide bonds in GOD itself. The appearance of $\mathrm{N} 1 s$ signal at the BE of about $399.2 \mathrm{eV}$ (Fig. 3d) also indicated that GOD had been covalently immobilized on the Si-PGMA surface. The Si-PGMA surface became more hydrophilic after GOD immobilization and the contact angle of the Si-PGMA-GOD surface decreased gradually to $53^{\circ}$ (Fig. 5) with the GOD immobilized time.

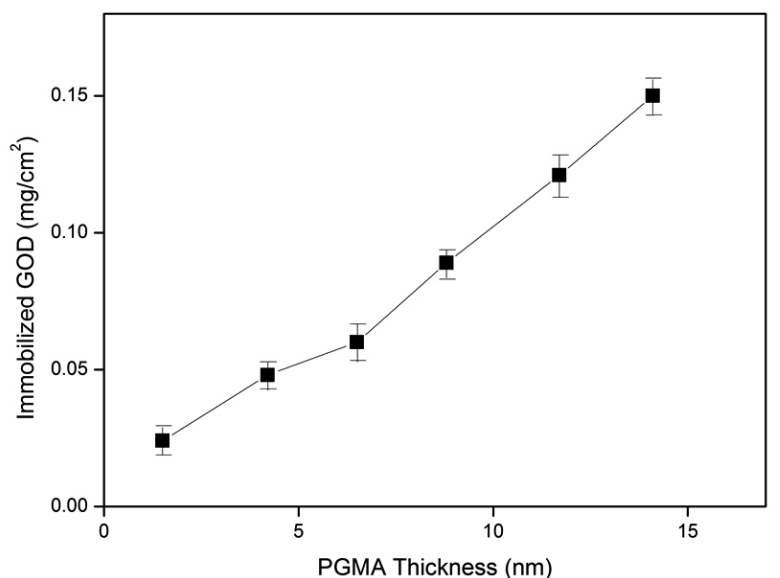

Fig. 6. Dependence of the amount of immobilized GOD on the thickness of the grafted PGMA (GOD immobilization time $5 \mathrm{~h}$ ). The starting surface corresponds to the Si-PGMA surface with the different thickness of PGMA.

The amount of immobilized GOD can be determined as the weight of immobilized GOD per area of the Si-PGMA-GOD surface, which is measured using the protein-dye interaction method. ${ }^{27,28}$ The amount of immobilized GOD (Fig. 5) also increased with the GOD immobilization time. When the immobilization time reached about 4-5 h, the surface concentration of immobilized GOD leveled off. It could be ascribed to the reaction between GOD and the epoxide groups in the top surface layer of the PGMA and the complete coverage of the Si-PGMA surface by GOD after about $4 \mathrm{~h}$. The further increase in the amount of immobilized GOD probably involved the immobilization of the enzyme in the subsurface region. ${ }^{14}$ As shown in Fig. 6, the amount of immobilized GOD increased with the increase in the thickness of the grafted PGMA layer on the Si-PGMA surface. Though steric hindrance from the thicker PGMA chains on the silicon surface increased, a large amount of GOD can be readily immobilized because of the high density of epoxide groups on the Si-PGMA surface, compared to the amount of GOD immobilized on polymer films via amide linkage, ${ }^{33}$ adsorption, ${ }^{34}$ and reversible immobilization. ${ }^{35}$

The storage stability is an important advantage of immobilized enzymes over the free enzymes, because the free enzymes lose their activities quickly. The stability of the immobilized GOD on the Si-PGMA-GOD surfaces, obtained from $25 \mathrm{~h}$ of RAFT polymerization and subsequently $5 \mathrm{~h}$ of GOD immobilization, was examined after the Si-PGMA-GOD substrates were stored at $4{ }^{\circ} \mathrm{C}$ for 14 days in air and in PBS solution, respectively. The relative XPS intensities of the nitrogen signal $([\mathrm{N}] /[\mathrm{C}]$ ratios) on the two surfaces remained almost unchanged compared to that of the freshly prepared Si-PGMA-GOD surface. And the water contact angles of the above two surfaces were about $53^{\circ}$ and $52^{\circ}$, respectively, which were comparable to that of the as-prepared Si-PGMA-GOD surface. The immobilized GOD molecules and the free GOD molecules from the commercial preparation retained about $90.5 \pm 3.1 \%$ and $80.2 \pm 2.8 \%$ of their original activity under both storage conditions, respectively. The result indicated that the immobilized GOD showed an improved stability than the free enzyme. Because the active conformation of the enzyme through covalent immobilization is stabilized by multipoint bond formation between the substrate and the enzyme molecules, covalent immobilization often result in the highest stabilization effect on enzyme activities..$^{36,37}$ Moreover, the stability of immobilized GOD also depends on the coupling method. For example, the stability of the immobilized enzyme via a 1,3-bifunctional aromatic coupling agent was reduced, which may results from the conformation deformation of the active enzyme and the loss in the multipoint binding ability of the enzyme ${ }^{37,38}$ As presented in this work, such a problem can be avoided to a large extent through the direct coupling between nucleophilic $-\mathrm{NH}_{2}$ groups of GOD with the epoxide groups of PGMA.

\section{CONCLUSIONS}

In summary, well-defined functional polymer brushes of GMA have been successfully grafted from silicon surface by surface-initiated RAFT-mediated process. The kinetic study revealed an approximately linear increase in thickness of the grafted PGMA brushes on the surfaces with polymerization time, which indicated that chain growth was consistent with a controlled process. GOD 
could be covalently immobilized on the modified silicon surface through the ring-opening reaction between the amine groups of the GOD and the epoxide groups of the grafted GMA polymer chains. The GOD-functionalized silicon hybrids could be used in silicon-based glucose biosensors

\section{ACKNOWLEDGEMENTS}

The work is supported by National Natural Science Foundation of China (20904044) and the New Century Excellent Talents in University of Ministry of Education of China (NCET-10-0157).

\section{REFERENCES}

1. S. E. Letant, B. R. Hart, S. R. Kane, M. Z. Hadi, J. G. Reynolds, $A d v$. Mater. 16, 689, (2004).

2. W. Cai, J. R. Peck, D. W. Weide, R. J. Hamers, Biosens. Bioelectron. 19, 1013, (2004).

3. K. C. Popat, T. A. Desai, Biosens. Bioelectron. 19, 1037, (2004).

4. S. Sharma, R. W. Johnson, T. A. Desai, Langmuir 20, 348, (2004).

5. S. T. Milner, Science 251, 905. (1991)

6. A. Halperin, M. Tirrell, T. P. Lodge, Adv. Polym. Sci. 100, 31, (1992).

7. K. Kato, E. Uchida, E. Kang, Y. Uyama, Y. Ikada, Prog. Polym. Sci. 28, 209, (2003).

8. P. T. Hurley, A. E. Ribbe, J. M. Buriak, J. Am. Chem. Soc. 125, 11334, (2003).

9. A. B. Sieval, A. L. Demirel, J. W. M. Nissink, M. R. Linford, J. H. Van der Mass, W. H. Dejeu, H. Zuilhof, E. J. R. Sudholter, Langmuir 14, $1759,(1998)$.

10. W. J. Royea, A. Juang, N. S. Lewis, Appl. Phys. Lett. 77, 1988, (2000).

11. C. J. Hawker, A. W. Bosman, E. Harth, Chem. Rev. 101, 3661, (2001).

12. L. Li, X. Yang, F. Liu, J. Shang, G. Yan, W. Li, J. Chil. Chem. Soc. 54, 397, (2009).

13. M. Baum, W. J. Brittain, Macromolecules 35, 610, (2002).

14. F. J. Xu, Q. J. Cai, Y. L. Li, E. T. Kang, K. G. Neoh, Biomacromolecules 6, 1012, (2005)

15. S. Edmondson, V. L. Osborne, W. T. S. Huck, Chem. Soc. Rev. 35, 14, (2004).

16. M. Ejaz, K. Ohno, Y. Tsujii, T. Fukuda, Macromolecules 33, 2870, (2000).

17. H. Liu, C. T. Mahony, F. Audouin, C. Ventura, M. Morris, A. Heise, Macromol. Chem. Phys. 213, 108, (2012).

18. Y. Wei, D. Z. Gao, L. Li, S. M. Shang, Polymer 52, 1385, (2011).

19. I. S. Altarawneh, V. G. Gomes, M. H. Srour, J. Appl. Polym. Sci. 114, 2356, (2009).

20. Z. P. Cheng, X. L. Zhu, G. D. Fu, E. T. Kang, K.G. Neoh, Macromolecules 38, 7187, (2005).

21. D. Chikhaoui-Grioune, A. Aqil, A. M. Zalfen, A. Benaboura, C. Jerome, J. Appl. Polym. Sci. 117, 1005, (2010).

22. F. Simal, A. Demonceau, A. F. Noels, Angew. Chem. Int. Ed. 38, 538, (1999).

23. Q. Peng, D. M. Y. Lai, E. T. Kang, K. G. Neoh, Macromolecules 39 , 5577, (2006).

24. S. Nishiyama, A. Goto, K. Saito, K. Sugita, M. Tanmada, T. Sugo, T. Funami, Y. Goda, S. Fujimoto, Anal. Chem. 74, 4933, (2002).

25. A. W. Eckert, D. Grobe, U. Rothe, Biomaterials 21, 441, (2000)

26. F. J. Xu, Z. L. Yuan, E. T. Kang, K. G. Neoh, Langmuir 20, 8200, (2004).

27. I. K. Kang, B. K. Kwon, J. H. Lee, H. B. Lee, Biomaterials 14, 787, (1993).

28. M. Bonde, H. Pontoppidan, D. S. Pepper, Anal. Biochem. 200, 195, (1993).

29. C. Bartholome, E. Beyou, E. B. Lami, P. Chaumont, F. Lefebvre, N. Zydowicz, Macromolecules 38, 1099, (2005).

30. J. F. Moulder, W. F. Stickle, P. E. Sobol, K. D. Bomben, Handbook of $\mathrm{X}$-ray Photoelectron Spectroscopy, Perkin-Elmer, Eden Prairie, MN, 1992.

31. P. Wang, K. L. Tan, E. T. Kang, K. G. Neoh, J. Mater. Chem. 11, 2951, (2001).

32. G. Beamson, D. Briggs, High-resolution XPS of Organic Polymer: the Scienta ESCA300 Database John Wiley, Chichester, UK, 1992.

33. N. Vasileva, T. Godjevargova, J. Membr. Sci. 239, 157, (2004).

34. M. Y. Arica, A. Denizli, T. Baran, V. Hasirci, Polym. Int. 46, 345, (1998).

35. M. Y. Arica, G. Bayramoglu, Biochem. Eng. J. 20, 73, (2004).

36. M. Arica, H. Yakup, J. Chem. Technol. Biotechnol. 58, 287, (1993).
37. C. C. Wang, G. H. Hsiue, J. Appl. Polym. Sci. 50, 1141, (1993).

38. J. C. Tiller, R. Rieseler, P. Berlin, D. Klemm, Biomacromolecules 3, 1021, (2002). 